\title{
A cross-sectional review of office and ambulatory blood pressure in children with renal diseases
}

\author{
Wun Fung HUI, Chon In KUOK, Winnie Kwai Yu CHAN \\ Department of Paediatrics, Queen Elizabeth Hospital, Hong Kong SAR
}

Background and Aims: Ambulatory blood pressure monitoring (ABPM) is an important tool for evaluation of blood pressure (BP) among high risk children including those with renal diseases. Our study aims to review the office BP and reports of ABPM performed in children with various renal conditions.

Method: We performed a cross-sectional review of the office BP records and ABPM reports of patients followed up in the paediatric nephrology clinic of Queen Elizabeth Hospital, Hong Kong from year 2013-2017. The diseases categories included congenital anomalies of kidney and urinary tract (CAKUT), glomerular diseases, cystic kidney diseases, renovascular hypertension, neurogenic bladder / bladder dysfunction and other conditions.

Results: Table 1 showed the demographic data and clinical characteristics of the 67 patients enrolled. Altogether $62.7 \%$ of subjects had ABPM hypertension (ABPM systolic or diastolic hypertension in either wake or sleep periods), and $40.3 \%$ of subjects had nocturnal dipping $<10 \%$ (non-dippers) (Table 2). Elevated blood pressure and hypertension by office BP were found in $21.9 \%$ and $35.9 \%$ of subjects respectively. There were $25 \%$ of subjects having masked hypertension (Figure). The highest ABPM indices and BP loads were found among those with renovascular hypertension, while those with CAKUT, glomerulopathy and neurogenic bladder / bladder dysfunction had significantly higher diastolic than systolic BP indices or load in both wake and sleep period $(p<0.05)$.

Distribution of hypertension category

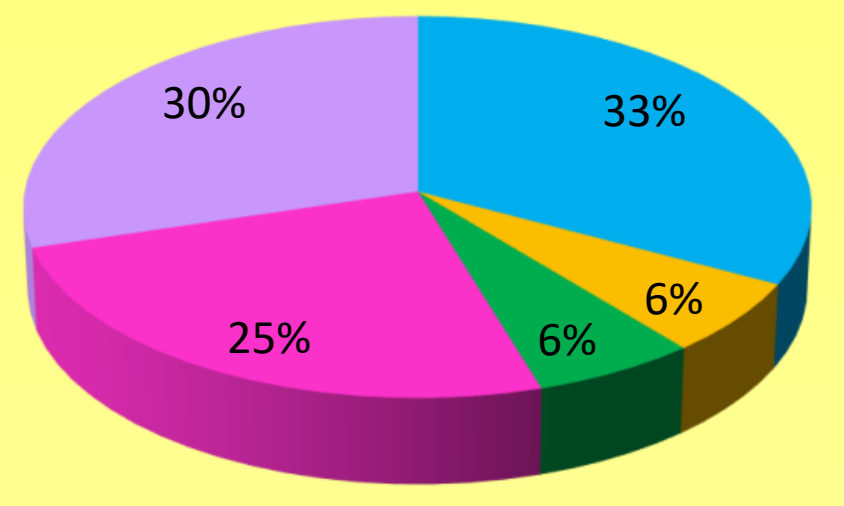

No hypertension Prehypertension White coat hypertension Masked hypertension Ambulatory hypertension
Table 1: Baseline characteristics of the study population

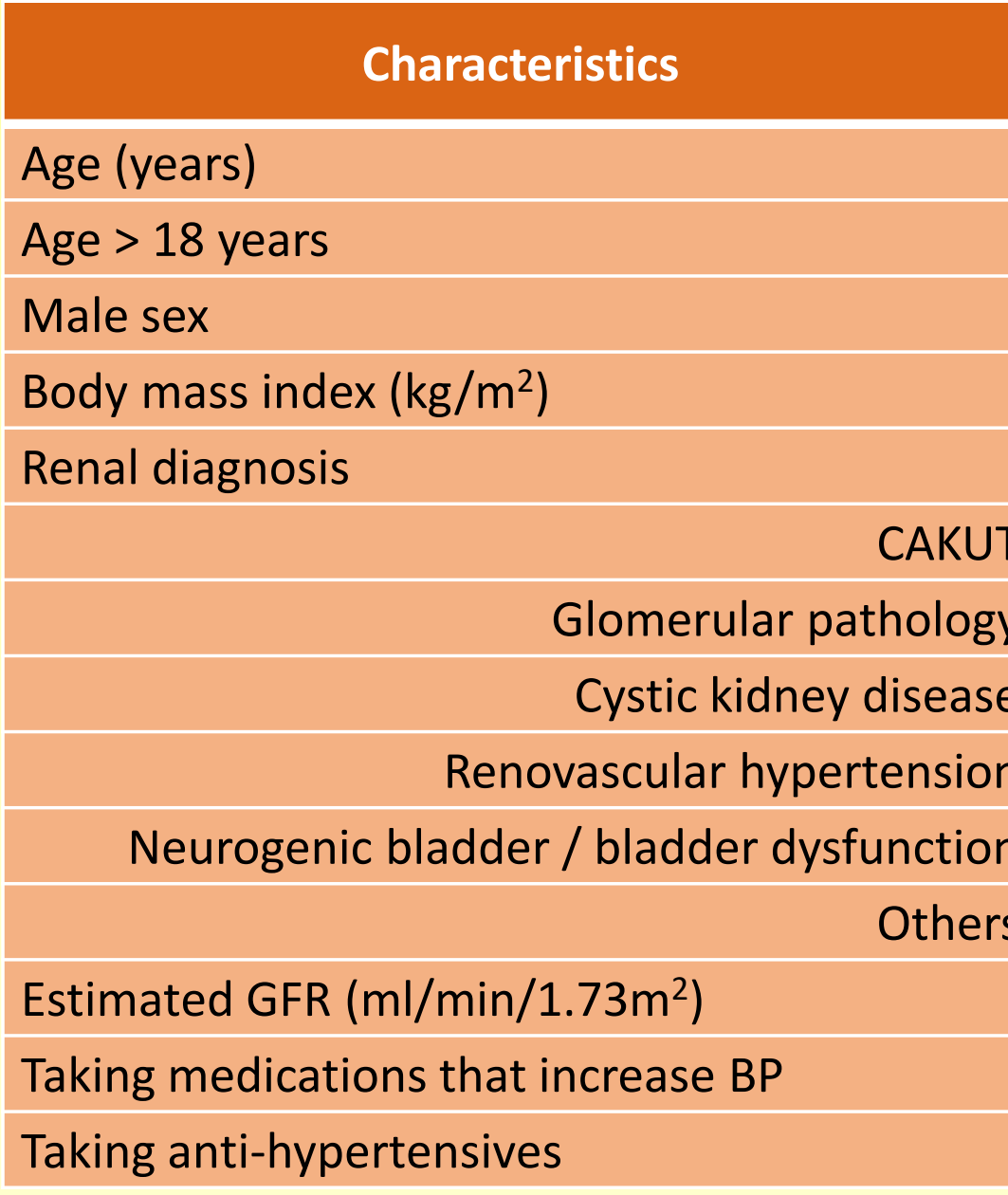

Table 2: Ambulatory blood pressure monitoring parameters

\begin{tabular}{|c|c|c|}
\hline & Characteristics & Values \\
\hline & Systolic BP index\# & $0.89(0.84,0.94)$ \\
\hline & Diastolic BP index\# & $0.90(0.80 .0 .97)$ \\
\hline What & Systolic BP load (\%)^ & $5.1(0.0,25.0)$ \\
\hline wake & Diastolic BP load (\%)^ & $16.1(3.1 .37 .2)$ \\
\hline & ABPM systolic BP hypertension & $17(25.4 \%)$ \\
\hline & ABPM diastolic BP hypertension & $23(34.3 \%)$ \\
\hline & Systolic BP index\# & $0.88(0.84,0.97)$ \\
\hline & Diastolic BP index $\#$ & $0.91(0.85,1.02)$ \\
\hline Clono & Systolic BP load (\%)^ & $5.6(0.0,25.0)$ \\
\hline sleep & Diastolic BP load (\%)^ & $25.0(7.1,50.0)$ \\
\hline & ABPM systolic BP hypertension & $19(28.4 \%)$ \\
\hline & ABPM diastolic BP hypertension & $34(50.7 \%)$ \\
\hline ABPM & ertension ${ }^{\S}$ & $42(62.7 \%)$ \\
\hline Noctu & dipping (\%) & $11.3(8.5,16.6)$ \\
\hline Non-d & & 27 (40.3\%) \\
\hline
\end{tabular}

Table 3: ABPM characteristics among children with different conditions

$$
\begin{gathered}
14.5(11.8,17.2) \\
4(6 \%) \\
42(62.7 \%)
\end{gathered}
$$

$19.3(17.6,22.6)$

$28(41.8 \%)$

$16(23.9 \%)$

$4(6.0 \%)$

$5(7.5 \%)$

$9(13.4 \%)$

$5(7.5 \%)$

$96.3(77.0 .111 .6)$

$8(11.9 \%)$

$21(31.3 \%)$
Neurogenic

Renovascular
hypertension
$\quad(n=5)$

bladder /

bladder

dysfunction
Age (years)

BMI $\left(\mathrm{kg} / \mathrm{m}^{2}\right)$

Taking anti-hypertensives

Wak
Sleep
Non-

Wake
Sleep

Systolic BP index

Diastolic BP index ${ }^{\#}$

Systolic BP load (\%)^

Diastolic BP load (\%)^

Systolic BP index\#

Diastolic BP index

Systolic BP load (\%)^

Diastolic BP load (\%)

\begin{abstract}
$13.4(10.0,16.4)$
\end{abstract}
$15.3(13.3,18.2)$

$13.6(9.4,14.8)$

$14.9(12.9,17.8)$

\section{ABPM hypertension ${ }^{\S}$}

$19.0(15.7,22.1)$

$19.9(18.6,23.8)$

$9(56.2 \%)$

$19.9(14.6,22.8)$

$1(25 \%)$

ABPM parameters

$0.88(0.83,0.93) \quad 0.87(0.83,0.96) \quad 0.84(0.79,0.85)$

$0.86(0.78,0.95) \quad 0.90(0.84,1.00) \quad 0.80(0.78,0.88)$

$3.1(0.0,15.1)$

$4.7(2.6,26.9)$

$0.86(0.83,0.90)$

$0.91(0.82,0.97)$

$0.0(0.0,17.6)$

$18.4(6.7,35.8)$

$$
11(39.3 \%)
$$

14 (50.0\%)
$7.8(0.0,38.8)$

$19.3(5.9,46.9)$

$0.88(0.83,1.01)$

$0.92(0.87,1.01)$

$4.8(0.0 .38 .7)$

$27.5(10.1,66.7)$

\begin{abstract}
$6(37.5 \%)$
\end{abstract}
11 (68.8\%)
$1.4(0.0,4.5)$

$6.4(1.3,14.9)$

$0.91(0.88,0.93)$

$0.93(0.84,1.02)$

$11.2(2.6,17.4)$

$20.3(3.0,45.6)$

\begin{abstract}
4 (100\%)
\end{abstract}
2 (50.0\%)
$17.9(17.3,26.7)$

$3(60.0 \%)$

$1.02(0.95,1.08)$

$1.04(0.96,1.16)$

$57.5(20.3,86.8)$

$67.5(45.0,76.7)$

$1.00(0.97,1.04)$

$1.09(0.96,1.18)$

$50.0(27.7,66.7)$

$66.7(50.0,70.6)$

\begin{abstract}
$2(40.0 \%)$
\end{abstract}
5 (100\%) $(n=9)$

$15.7(13.4,17.4)$

$19.0(15.7,22.9)$

$1(11.1 \%)$

$14.8(13.3,20.0)$

$21.1(19.1,31.4)$

$3(60.0 \%)$

Others $(n=5)$

p-value

0.26

0.57

0.02

\#: BP Index is defined as average BP values divided by the respective $95^{\text {th }}$ percentile values $/{ }^{\wedge}$ : BP load is defined as percentage of readings that exceeds the respective $95^{\text {th }}$ percentile values

$*$ : Non-dippers: nocturnal dipping <10\% / §: ABPM hypertension: any ABPM systolic or diastolic BP hypertension in wake or sleep period

All values were expressed in either median $\left(25^{\text {th }}\right.$ percentiles, $75^{\text {th }}$ percentiles) or number $(\%)$

Conclusion: Masked hypertension and non-dippers were common among children with kidney diseases. The highest ABPM indices and BP loads were

found among children with renovascular hypertension. Children with CAKUT, glomerulopathy and neurogenic bladder / bladder dysfunction also had significantly higher diastolic than systolic BP indices or load. ABPM should be recommended for children with renal conditions. 